\title{
Effect of fillers on water and chemical absorption behavior of natural fiber reinforced epoxy composites
}

\begin{abstract}
Natural fiber is gaining more interest in these days due to its low cost, less weight and density that is make the natural fiber more attractive. Present work demonstrates jute fiber is used as reinforcement material and epoxy is used as a matrix material. And cenosphere is used as filler or secondary reinforcing material which will add better properties the jute fiber/epoxy composite. Various proportions of cenosphere is added in the composite to characterize the water and chemical absorption behavior and thickness swelling behavior of the composites. Water absorption tests was carried out for three different water medium and chemical absorption test was performed for two different chemicals. The composite samples were kept for long interval of time to analyze the absorption behavior.
\end{abstract}

Keywords: cenosphere, jute fabric, water absorption, chemical absorption, filler, natural fiber
Volume 7 Issue 3 - 202I

\author{
Sachin KO,' Maruthi BH,' Puttaswamaiah S,' \\ Satish Babu ${ }^{3}$ \\ 'Department of Mechanical Engineering, East West Institute of \\ Technology, India \\ ${ }^{2}$ Department of Mechanical Engineering, Presidency University \\ Bangalore, India
}

\author{
Correspondence: Sachin KO, Department of Mechanical \\ Engineering, East West Institute of Technology, Bangalore, India, \\ Email k.o.sachin@gmail.com
}

Received: April 14, 202I | Published: May 03, 202 I

\section{Introduction}

Due to low cost, light weight, high specific modules, renewability and biodegradability, natural fiber-reinforced composite materials receive great responsiveness in structural applications, automotive industries and household applications. ${ }^{1}$ Through holding their relative position, the matrix layer surrounds and maintains the reinforcement materials. The strengthening impart their excellent mechanical and physical properties in order to increase the matrix properties. ${ }^{2,3}$ The main reasons for popular growth of natural fiber composites are the easily availability in large and of good quality of wide range of fibers, and are sustainable. ${ }^{4}$ Natural fibers application across the world, also given the importance on bio-materials, bio-composite materials based on bio epoxy resin. ${ }^{1}$ Cenosphere is a ceramic-abundant industrial waste generated in thermal power plants during coal burning. The influence of cenosphere as filler on the involuntary actions in biocomposites constructed from bamboo. Mechanical and heat related properties of composites made from high density polyethylene (HDPE) - fly ash cenosphere. Cenosphere is largely used to slash the mass of extensively used plastics, rubbers, resins, etc. Present work highlights, the composite materials are primary reinforced and secondary reinforced with cenosphere as filling material with epoxy resin. The absorption behavior of the composites is evaluated to know the influence of various percentage of cenosphere.

\section{Materials and experimentation}

The materials used for this present work:

\section{Jute fabric \\ II. Cenosphere}

III. Epoxy resin (L-12 and K-6)

In this work, jute fabric is used as a reinforcement material which is extracted from the stem fiber plant. Because of its impressive firmness and stiffness, jute fibers are especially fit for manufacturing of coarse fibers, stable and durable and fabrics. Cenosphere are insubstantial, inert, and hollow spheres has a main constitute of silica and alumina, are packed with air or gases, and are derivatives of the combustion of pulverized coal at the thermal power plants. Epoxy resin Lapox-12 (L12 ) is used as matrix material and for this a suitable hardener K-6 is used (Table 1). The composite sheets were designed using hand layup technique. As per ASTM specifications, the composite board is treated according to normal measurements. The proportion of filler material was to be measured in all of the composite boards as shown in Figure 1. These composite specimens/samples are investigated to know the water absorption and chemical absorption behavior. And also to know the effects of absorption on the inter-facial bonding between fibers and thermoset matrix evaluating the durability of the composites.

Table I Various proportions in composites

\begin{tabular}{ll}
\hline Volume fraction & Code \\
\hline Jute Fabric $60 \%+$ Epoxy $40 \%+$ Cenosphere $0 \%$ & JEC I \\
Jute Fabric $50 \%+$ Epoxy $40 \%+$ Cenosphere 10\% & JEC 2 \\
Jute Fabric $45 \%+$ Epoxy $40 \%+$ Cenosphere 15\% & JEC 3 \\
\hline
\end{tabular}

\section{Water absorption}

Water absorption test was conducted according to ASTM D 5229 standard. Specimens are observed, and values were recorded at regular interval of time. Composite samples were dried in an oven at $70^{\circ}$ Celsius for 24 hours, weighed and then dripping in a bath of normalwater and seawater at room temperature. This is to know the effect of absorption of water through micro pores present and the effect on composites which will directly affect the durability of the composites. For every 24 hours, samples were taken off from the water, dried with a cotton cloth and weighed again till samples attain saturation.

\section{Chemical absorption}

Chemical absorption test was conducted according to ASTM D 543 standard depending on environmental conditions. A heavy chemical absorption and low dimensional flexibility are a significant drawback in utilizing natural fibers in enduring composite application. 
Fiber swelling will load composite micro-cracking and mechanical properties degradation. This issue can be overcome by chemically treating fibers to decrease the hydroxyl group on the surface of the fibers. Strong intermolecular bonding of the fiber-matrix reduces the amount of chemical-absorption in bio composite. The fiber surface must be washed and chemically adjusted, and the surface roughness must be improved to enhance the interface adhesion. For every 24 hours, the specimens were taken off from the Sulphuric acid and Sodium hydroxide dried with a cotton-cloth and weigh up again till samples attain saturation.

\section{Results and discussion}

\section{Water absorption}

For these tests, the specimen was cut into size of $50 \times 50 \times 10 \mathrm{~mm}$ as per standards and experiments are conducted. For moisture absorption test, the specimens are dipped two types of i.e. normal water and sea water. The weight and thickness of the specimen is checked each day and noted. It is noted for about 14 days until it attains saturation. Water, predominantly absorbed at the fiber interface and matrix. As evident from Figure 1 and Figure 2 shows, that water content of the composite increased with time and became a constant. It is found that specimens without filler absorbed more water when compared to cenosphere filled composites in both normal and sea water. It was also observed that, normal and sea water was absorbed continuously till $10^{\text {th }}$ day and further the curve was fattened means composite samples attained saturation. Cenosphere filled composites are more repulsive than with no filler composite which indicated that, the interstitial positions or pores within the composites are filled with these fillers and do not allow water to fill or cover that pores. So in cenosphere filled composites absorption is less. From water thickness swelling results i.e. from Figure $3 \&$ Figure 4 graphs it was noticed that samples in normal water and sea water does not have different scale of swelling means absorption is almost same. In both normal water and sea water, the samples with and without filler continuously absorb water till $10^{\text {th }}$ day and attains saturation till $14^{\text {th }}$ day. Here also filled composites have better resistance to absorption compared to unfilled composites. Hence, $15 \%$ cenosphere filled composites exhibits better water absorption and thickness swelling behavior. This property evaluation helps us to understand about the less normal and sea water absorption in filled composite behavior for further applications.

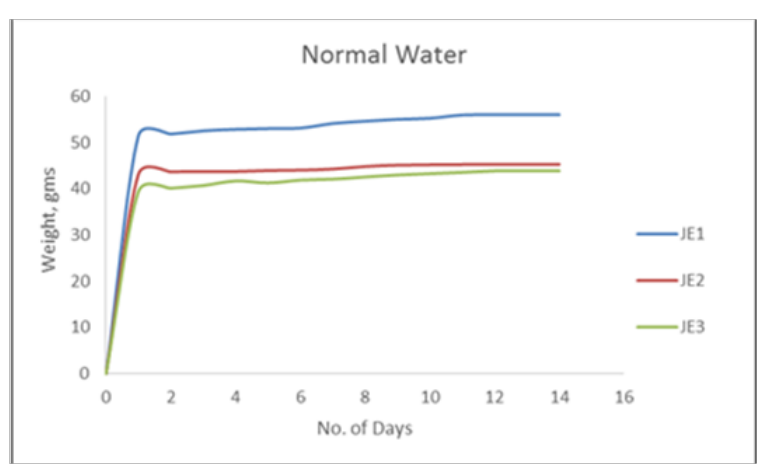

Figure I Shows the graphs for water absorption of various composites in normal water.

\section{Chemical absorption}

For these tests, the specimen was cut into size of $50 \times 50 \times 10 \mathrm{~mm}$ as per standards and experiments are conducted. For chemical absorption test, the specimens are dipped in 2 types of chemical sulfuric acid and sodium hydroxide. The two different chemical are based on acidic and base in nature. The weight and thickness of the specimen is checked each day and noted. It is noted for about 12 days till it attains saturation. Chemical absorption test was done to compare the behavior of with to without cenosphere filled composites. These chemicals based on acidic and base (alkaline) nature affects the physical behaviors of these composites are shown from Figure $5 \&$ Figure 6. With filler and without filler composite samples were dipped in sulphuric acid and sodium hydroxide for 11 days. It was observed that, till 8th day the chemical was absorbed and weight was gained but in sulphuric acid due to acidic nature the samples ruptured and sudden weight loss was observed. And also we can see from the graphs that, $15 \%$ cenosphere filler composite sample showed resistance to absorption of this chemical when compared. In sodium hydroxide, samples absorb till 8th day and weight gain was found. Further attains saturation till 11th day. Here also filled composite samples cover the pores or the interstitial position in the samples affect the accumulation of chemicals resulting less absorption when compared to cenosphere unfilled composite sample.

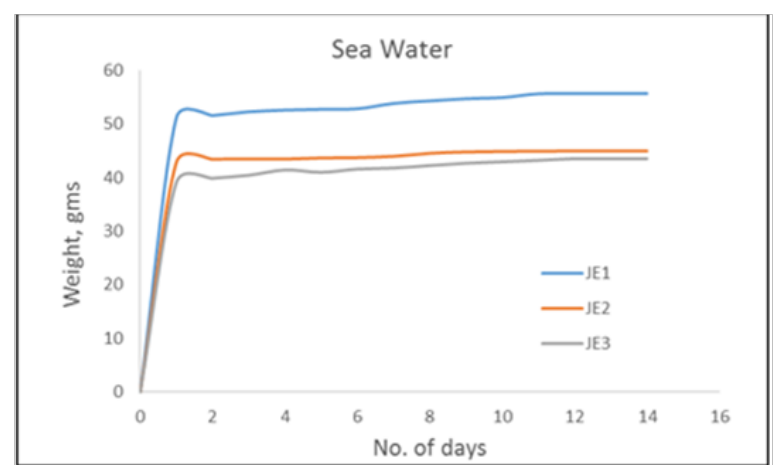

Figure 2 Shows the graphs for water absorption of various composites in sea water.

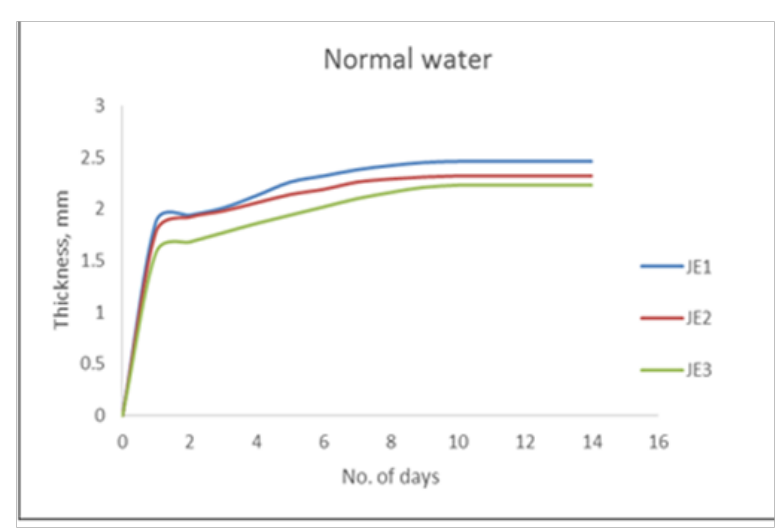

Figure 3 Shows the graphs for thickness swelling of various composites in normal water.

From chemical thickness swelling results i.e. from Figure $7 \&$ Figure 8 graphs it was depicts that samples in sulphuric acid and sodium hydroxide does not have different scale of swelling means absorption is almost same. In sulphuric acid, the sample with and without filler continuously absorb water till $8^{\text {th }}$ day and further sample distorted due to the acidic nature of sulphuric acid. And loss in chemical thickness swelling was observed. But $15 \%$ cenosphere filled composite was more stable compared to other two samples. In sodium hydroxide, we found the usual nature of chemical absorption and attaining saturation which indicates the base or the alkaline nature of chemical has less effect on the composite samples. Also it was found 
that, cenosphere filled composites have better resistance to absorption compared to unfilled composites. Hence, $15 \%$ cenosphere filled composites exhibits better chemical absorption and thickness swelling behavior. This property evaluation helps us to understand about how the chemical i.e. sulphuric acid and sodium hydroxide affects the cenosphere filled composite behavior for further applications such as chemical storage tanks or chemicals spilled out on automotive components.

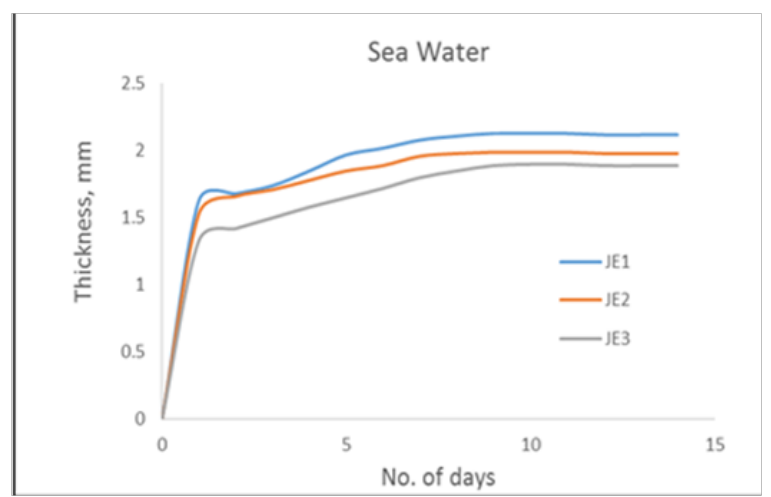

Figure 4 Shows the graphs for thickness swelling of various composites in sea water.

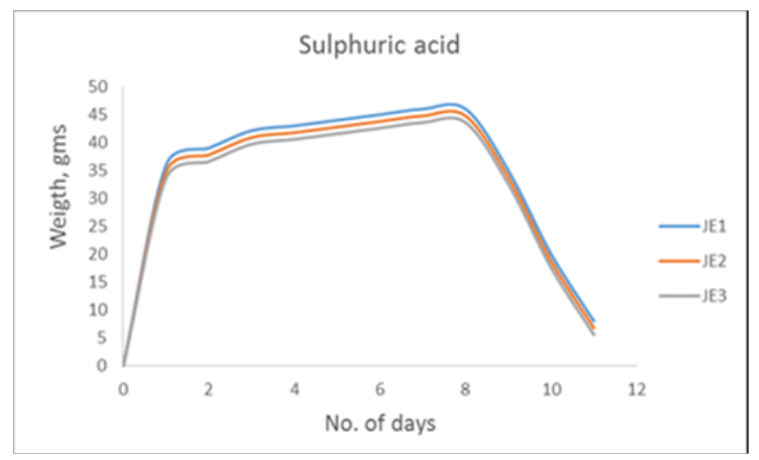

Figure 5 Shows the graphs for chemical absorption of various composites in sulphuric acid.

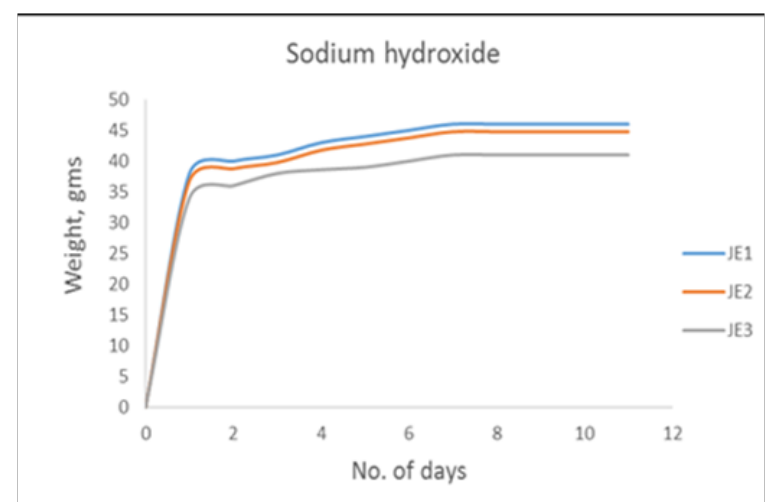

Figure 6 Shows the graphs for chemical absorption of various composites in sodium hydroxide.

\section{Conclusion}

Based on the absorption study, water and chemical absorption tests and also, water and chemical thickness swelling tests were conducted with composition of jute fabric, cenosphere and epoxy resin composites. These particulates are spotted to be same size and the specific surface area of the cenosphere is quite low. The addition of cenosphere enhances the water absorption resistance of the composites, but it depends upon the amount of cenosphere up to $15 \mathrm{wt} \%$. More weight gain and thickness swelling percent is greater in normal water as compared to sea water on filler loading. Sulphuric acid ruptures the composite sample and in sodium hydroxide has less effect on the composite samples. ${ }^{5-12}$

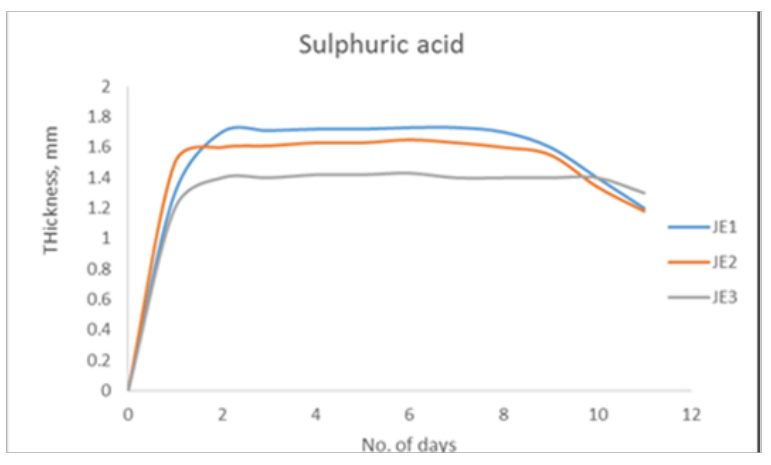

Figure 7 Shows the graphs for chemical thickness swelling of various composites in sulphuric acid.

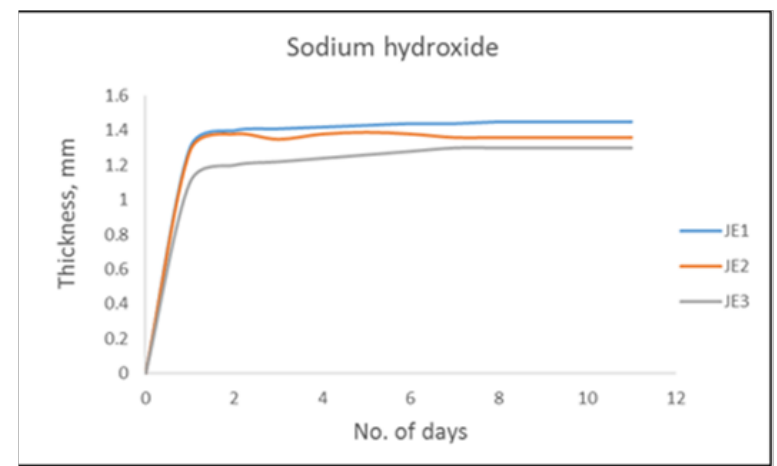

Figure 8 Shows the graphs for chemical thickness swelling of various composites in sodium hydroxide.

\section{Acknowledgements}

None.

\section{Conflicts of interest}

The authors declare that there is no conflict of interest.

\section{References}

1. Chandramohan D, Marimuthu K.AReview of natural fibers. International Journal of Recent Research and Applied Studies. 2011;8(2):194-206.

2. Irimia-Vladu M. Green electronics: Biodegradable and biocompatible materials and devices for sustainable future. Chem Soc Rev. 2014;43:588-610.

3. Kim DH. Dissolvable films of silk fibroin for ultrathin conformal biointegrated electronics. Nature Mater. 2010;9:511-517.

4. Janu Sharma, Navin Chand. Effect of cenosphere on dielectric properties of low density polyethylene. Physic. 2012;2:26-33.

5. Soma Dalbehera, SK Acharya. Effect of cenosphere addition on the mechanical properties of jute-glass fiber hybrid. $J$ epoxy composites. 2015:1-12.

6. Hemalata Jena, Mihir Kumar Pandit. Effect of cenosphere on mechanical properties of bamboo-epoxy composites. Journal of Reinforced Plastics and Composites. 2013:794-801. 
7. Manohara V, Sreenivasa CG, Bharath KN. Evaluation of Tensile Behavior of Sea Shell Jute Fabric Reinforced Composite. International Journal of Advanced Research in Mechanical and Production Engineering and Development. 2014;1:142-147.

8. Kolay PK, Singh DN. Physical, chemical, mineralogical, and thermal properties of cenospheres from an ash lagoon. Cement and Concrete Research. 2001;1:539-542.

9. Girish SK, Devendra, Bharath KN, Effect of Sodium bicarbonate on Fire behaviour of filled E-Glass Reinforced Epoxy Composites. IOP Conf. Series : Materials Science and Engineering. 2016;149:1-6.
10. Deepthi MV, Madan Sharma. Mechanical and thermal characteristics of high density polyethylene-fly ash Cenosphere composites. Materials and Design. 2010;31:2051-2060.

11. Sivasaravanan S, Bupesh Raja VK. Impact Characterization of Epoxy LY556/E-Glass Fibre/ Nano Clay Hybrid Nano Composite Materials. 2014;97:968-974.

12. Imoisili PE, Ukoba KO. Effect of Filler Volume Fraction on the Tensile Properties of Cocoa-Pod Epoxy Resin Composite. International Journal of Science and Technology. 2012;2:432-443. 